\title{
Effets de l'environnement social au cours du développement sur l'apparition et l'intensité de l'activité sexuelle du jeune bélier
}

\author{
P. ORGEUR, G. VENIER et J.P. SIGNORET \\ I.N.R.A., Station de Physiologie de la Reproduction \\ Laboratoire du Comportement animal \\ Centre de Recherches de Tours, F 37380 Monnaie
}

\section{Résumé}

Deux séries expérimentales successives montrent que de jeunes béliers de race Ile-de-France, séparés des femelles depuis le sevrage à l'âge de 3 mois, présentent un retard dans l'apparition de l'activité sexuelle lors de la puberté lorsqu'on les compare à des mâles restés en présence de jeunes agnelles. Ce défjcit concerne aussi bien le taux de jeunes mâles inactifs en présence de femelles en estrus que la possibilité d'effectuer des collectes de sperme.

Par contre, l'absence de la mère, consécutive à un élevage en allaitement artificiel, et la ségrégation sexuelle précoce sont sans conséquences notables dans la mesure où les jeunes mâles sont entretenus en présence de jeunes femelles à partir du sevrage. Au contraire, si la ségrégation est maintenue, un retard important dans l'apparition de l'activité sexuelle est observé.

De même, l'isolement physique consécutif à un élevage en case individuelle dès la naissance n'entraîne pas de déficit dans la mesure où un contact avec des femelles est établi après le sevrage.

La présence de femelles pendant la période qui précède et avoisine la puberté - entre 3 et 6 mois - paraît donc nécessaire à la mise en place d'un comportement sexuel précoce et efficace qu'implique la mise en testage des futurs reproductcurs. puberté.

Mots clés : Ovins, comportement sexuel mâle, développement, environnement social,

\section{Introduction}

La capacité reproductrice de l'animal est conditionnée par de nombreux facteurs physiologiques, génétiques, nutritionnels, sanitaires, ainsi que par l'environnement (rythme lumineux, température). Son expression dépend de l'efficacité du comportement sexuel, que le mâle soit utilisé en accouplement naturel ou pour l'insémination artificielle. Des études éthologiques ont mis en évidence des perturbations notables du comportement sexuel à la suite de privations sociales plus ou moins précoces, telles 
que l'isolement, la privation du contact avec la mère ou la ségrégation des sexes. Ces troubles vont chez le mâle, d'une mauvaise organisation posturale (anomalies du comportement de monte) à des préférences homosexuelles très marquées et même à une inhibition parfois définitive des réactions sexuelles.

Dans le cas particulier des ovins, des études effectuées sur de très grands nombres de béliers font ressortir l'existence d'une proportion non négligeable de jeunes mâles «sexuellement inhibés»:

Ces animaux n'ont aucune réaction sexuelle lors de leur mise en présence avec des brebis en oestrus, bien qu'ils soient physiologiquement pubères. Cependant la plupart d'entre eux se révèle ultérieurement capable de s'accoupler mais avec un délai plus ou moins important, et leur aptitude à s'accoupler reste inférieure à celle des mâles qui se sont accouplés d'emblée (MatTner et al., 1971 a, 1973).

Si les conditions optimales d'une bonne croissance corporelle des jeunes béliers reproducteurs sélectionnés sont généralement réunies, il n’est pas tenu compte de l'influence des conditions sociales au cours de l'élevage sur les manifestations ultérieures du comportement sexuel.

C'est pourquoi nous avons entrepris une étude expérimentale de l'effet de l'environnement social au cours du développement sur la mise en place de l'activité sexuelle des jeunes béliers.

Nous avons envisagé :

- d'une part, les conséquences de l'absence de la mère et celles de la ségrégation sexuelle, ou même de l'isolement, précoces, grâce aux méthodes d'allaitement artificiel ;

- d'autre part, l'influence que pourrait avoir la présence de femelles entre le sevrage et la puberté.

Pour mesurer les conséquences de ces divers traitements sur l'apparition, l'évolution et le niveau de l'activité sexuelle, les caractéristiques du comportement sexuel ont été étudiées dans différentes conditions en accouplement naturel, et en collectes de semence au vagin artificiel dans l'optique de l'insémination artificielle.

\section{Matériel et méthodes}

\section{A. Animaux et conditions d'élevage}

125 agneaux de race Ile-de-France sont issus de reproduction programmée.

Leurs naissances groupées sur 3 ou 4 jours ont permis de former des groupes d'élevage aussi homogènes que possible. Ils ont toujours été entretenus en bergerie sur aire paillée.

Nous avons pris en considération 2 périodes successives du développement du jeune bélier. 


\section{Période d'allaitement}

Elle est comprise entre la naissance et le sevrage. ficiel.

Les agneaux ont été soit allaités par leur mère, soit soumis à un allaitement arti-

Ceux allaités par leur mère (Allaitement Maternel : A.M.) avaient accès à un parc adjacent où ils ont pu progressivement consommer un aliment de sevrage et du foin. Une séparation des mères pendant un temps qui s'est accru régulièrement a conduit à un sevrage définitif entre 90 et 110 jours (environ 15 semaines ou 3 mois et demi).

Ceux soumis à l'allaitement artificiel (A.A.) ont été séparés de leur mère après 24 à $48 \mathrm{~h}$ afin qu'ils puissent absorber du colostrum. Ils ont alors été regroupés par 25 dans une nurserie où la température n'était jamais inférieure à $15^{\circ} \mathrm{C}$. Du lait reconstitué, dilué et porté par une machine à une température de $35^{\circ}$ la première semaine puis de $20^{\circ}$, était mis à la disposition des agneaux grâce à des tétines en caoutchouc.

Ce régime alimentaire était progressivement remplacé par un aliment de sevrage additionné de condiments minéraux puis de foin. Au-delà de 7 à 8 semaines, ces agneaux n'ont plus consommé de lait.

Les sujets conservés pour la suite de l'expérience ont alors été choisis sur des critères zootechniques comme la croissance corporelle, les aplombs, l'état sanitaire.

\section{Période post-sevrage}

Depuis le sevrage jusqu'à l'âge d'environ 6 mois, les animaux ont été entretenus en groupes et alimentés de foin et concentré et de paille à volonté.

\section{B. Plan expérimental (fig. 1)}

La situation qui se rapproche le plus des conditions naturelles d'élevage en troupeau libre est celle où l'allaitement maternel est suivi d'un élevage en mixité jusqu'à la puberté. Ce traitement a été considéré comme témoin et répété dans les trois séries expérimentales.

\section{Expérience $I$}

Dans la période d'allaitement, tous les agneaux ont été élevés en groupes mixtes. Certains étaient allaités par leur mère (A.M.), d'autres en étaient privés (A.A.). Dans la période post-sevrage, une partic des mâles de chaque groupe a été maintenue en mixité ( 2 groupes de 11 mâles +6 femelles) ; l'autre partie a été privée de la présence de femelles ( 2 groupes de 11 mâles). Dans ce cas, les jeunes béliers ont été entretenus dans un bâtiment isolé de tout contact avec des femelles.

\section{Expérience $I I$}

Dans la période d'allaitement ou infantile, un groupe mixte a été élevé avec les mères. Un autre groupe, constitué uniquement de mâles, a été entretenu en allaitement artificiel. 


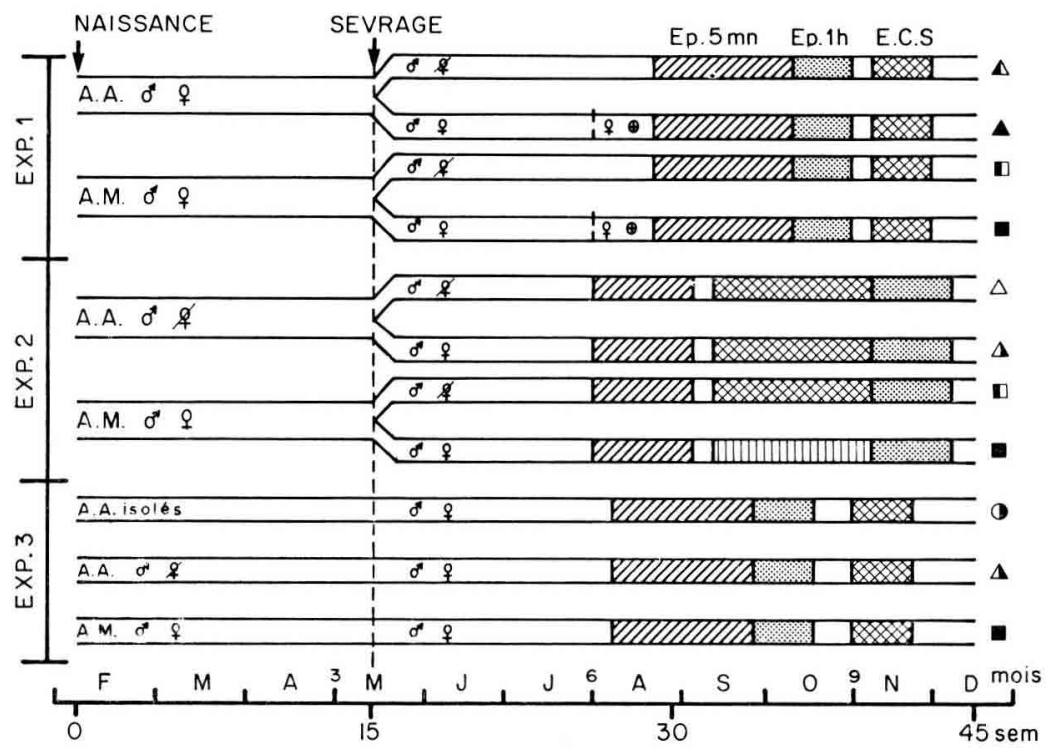

FIG. 1

Schéma du protocole expérimental.

Scheme of the experimental design.

A.M. : Allaitement maternel.

Dam reared animals.

A.A. : Allaitement artificiel.

Artificially reared animals.

\& : Mâles isolés de femelles.

Males only.

ô ㅇ : Mâles en présence de femelles.

Males and females.

$\hat{\sigma} \oplus$ : Femelles injectées de Benzoate d'Estradiol.

Females injected with Oestradiol benzoate.

Ep. $5 \mathrm{mn}$ : Epreuves de comportement sexuel de 5 minutes. 5 minutes sexual behaviour tests.

Ep. $1 \mathrm{~h}$ : Epreuves de comportement sexuel d'une heure. One hour sexual behaviour tests.

E.C.S. : Essais de collecte de semence.

Semen collection tests.

Pour l'ensemble des figures :

- le triangle signifie allaitement artificiel ;

- le carré signifie allaitement maternel ;

- le cercle, les animaux élevés dans l'isolement.

La présence de femelles dans l'une ou l'autre phase du développement est représentée par la couleur noire du sigle.

For all the figures :

- a triangle means artificially reared;

- square means dam reared;

- circle means reared in physical isolation.

The presence of the females during one and/or the other phase of the development is indicated by black in the sign. 
Dans la période post-sevrage ou prépubère, une partie des mâles de chaque groupe a été élevée en présence d'agnelles (1 groupe de 19 mâles +6 femelles et 1 groupe de 11 mâles +6 femelles); l'autre partie a été entretenue en groupes de mâles dans un bâtiment isolé ( 2 groupes de 11 mâles).

\section{Expérience III}

Dans la période infantile, des agneaux mâles ont été élevés en allaitement artificiel soit en groupe, soit isolés dans des cases individuelles. Dans ce dernier cas, ils pouvaient se sentir, s'entendre et éventuellement se voir à travers les claies en bois séparant leurs cases. D'autres ont été allaités par leur mère (A.M.) en présence d'agnelles de leur âge.

Après le sevrage, les 11 mâles élevés en cases individuelles ont été réunis et 3 femelles du même âge ont été introduites parmi eux, de même qu'avec les 12 allaités artificiellement en groupe. La présence d'agnelles a été maintenue dans le groupe issu d'A.M. ( 8 mâles +2 femelles) : groupe témoin.

Les femelles présentes dans les lots mixtes des différentes séries expérimentales, puis lors des épreuves ultérieures de comportement sexuel, ont été ovariectomisées à l'âge de 5 mois, avant leur puberté.

\section{Mesure de l'activité sexuelle}

Les jeunes béliers ont été mis à l'épreuve dans différentes conditions normalisées à partir de l'âge de 6 mois afin de mettre en évidence l'apparition, l'évolution et le niveau de l'activité sexuelle.

Dans l'expérience I, et dans celle-ci seulement, nous avons observé les réactions des mâles lors de l'apparition de la réceptivité sexuelle chez des agnelles présentes dans les deux groupes mixtes d'élevage. Ceci mime, en effet, les conditions naturelles.

Pour ceci, les jeunes agnelles ont été rendues sexuellement réceptives par traitement hormonal (injections de progestérone, puis de benzoate d'oestradiol, RoBjNson, 1954). La durée de la réceptivité, comme les réactions comportementales sont, dans ces conditions, comparables à celles de l'œstrus naturel. Dans chaque groupe mixte, deux des 6 agnelles ont ainsi été traitées au cours de 3 semaines successives.

Des observations associées à un système de marquage consistant à enduire la croupe des femelles avec un crayon gras spécial qui colorait le poitrail des mâles lorsqu'ils chevauchaient, nous ont permis d'évaluer l'activité de monte le jour de la réceptivité des femelles traitées.

Pour l'ensemble des animaux expérimentaux, la mesure de l'activité copulatoire a été effectuée par 3 types d'épreuves normalisées.

\section{Mise en évidence et évolution du comportement copulatoire (FABRE, 1977)}

Deux fois par semaine, chaque mâle a été mis, pendant 5 minutes, en présence de 4 femelles ovariectomisées dont 2 avaient reçu un traitement hormonal destiné à provoquer leur réceptivité sexuelle (RoBINSON, 1954). Dix à 14 épreuves ont été effectuées selon les séries expérimentales (fig. 1). 


\section{Capacités sexuelles}

Ce test était dérivé du précédent, mais sa durée était d'une heure et 6 femelles réceptives ont été proposées à chaque mâle, ceci 2 fois à 2 semaines d'intervalle.

Ces épreuves ont suivi celles de 5 minutes dans la première et la troisième série expérimentale. Dans la seconde, elles ont eu lieu en dernier, donc après les essais de collecte de semence (fig. 1).

\section{Collectes de semence}

Chaque mâle était mis pendant 3 minutes en présence d'une femelle sexuellement réceptive et en contention. Une tentative de collecte de sperme au vagin artificiel était effectuée à chaque monte du bélier.

Ces essais ont eu lieu 5 fois en 3 semaines après les tests de 5 minutes et d'une heure vers l'âge de 9 mois (Exp. I et III), et 13 fois réparties sur 7 semaines entre ces 2 séries d'épreuves (vers l'âge de 7 mois et demi), lors de l'exp. II (fig. 1).

\section{Eléments de comportement sexuel enregistrés. Analyses effectuées}

Les flairages anogénitaux, les approches ritualisées, souvent accompagnées d'un mouvement d'une patte antérieure et parfois d'une émission sonore, les montes avec ou sans mouvements pelviens et les accouplements ou les collectes de semence ont été comptabilisés.

L'analyse statistique a été effectuée sur :

- les montes, les accouplements et les collectes de semences : ce sont les actes qui caractérisent le mieux l'activité sexuelle des animaux (test non paramétrique de ManN \& Whitney : Siegel, 1956);

- le rapport du nombre d'accouplements au nombre de montes (efficacité sexuelle traduisant l'adaptation posturale nécessaire à la réalisation du coït) (test de MANN \& WHITNEY);

- le temps écoulé entre le début d'une épreuve et le premier accouplement (latence) (Test F);

- la relation entre le jeu sexuel post-natal et le comportement sexuel adulte (test de corrélation par rang de Spearman : Siegel, 1956).

\section{Résultats}

A. Effet de l'induction d'une réceptivité sexuelle des femelles dans les groupes mixtes

Le premier contact avec des agnelles sexuellement réceptives stimule considérablement l'activité sexuelle des jeunes mâles inexpérimentés, qu'ils aient été élevés en présence ou en l'absence de leur mère. Ainsi, le niveau d'activité devient dans les 2 groupes mixtes beaucoup plus important qu'avant le traitement et très nettement supérieur à celui des mâles des 2 groupes unisexués $(P<0,001$; fig. 2 a) avec lequel il était 
équivalent avant ce traitement. La grande majorité des approches ritualisées (82 p. 100) et des montes $(95$ p. 100) était dirigée vers les femelles $(P<0,001$; fig. 2 b), malgré leur nombre inférieur à celui des mâles (6 contre 10). Cette activité dirigée vers les femelles était orientée sélectivement vers celles traitées aux oestrogènes, qu'elles soient réceptives ou non $(91$ p. 100 des approches ritualisées et 94 p. 100 des montes; $\mathbf{P}<0,001$; fig. 2 c).

(a)

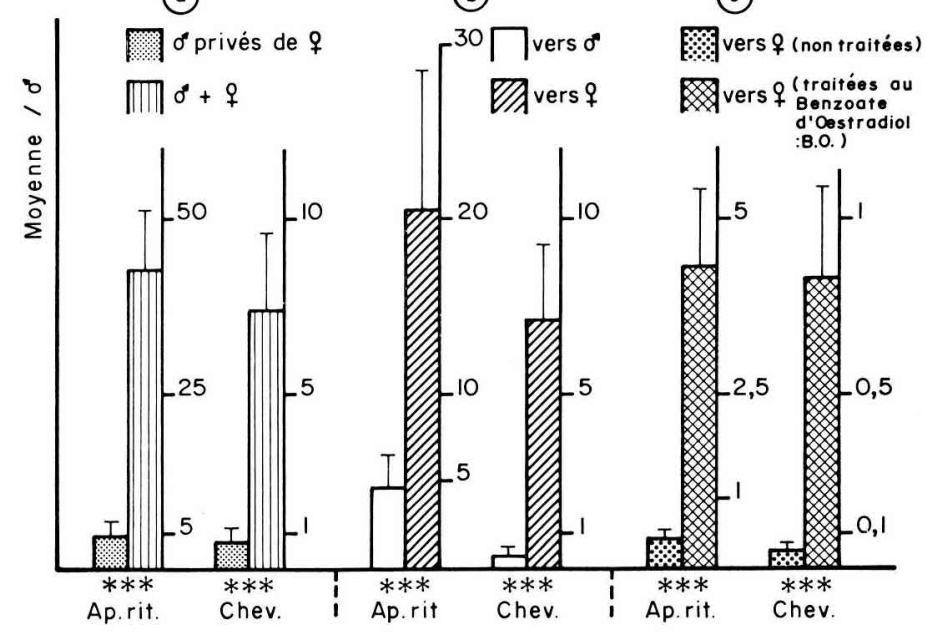

FIG. 2

Effets de l'induction hormonale de la réceptivité sexuelle chez les femelles des groupes mixtes (Exp. 1).

$* * *=\mathrm{P}<0,001$.

Effects of hormonal induction of sexual receptivity in the lamb ewes (mixed groups - Exp. 1).

Lors des contacts avec ces femelles traitées aux cestrogènes, 18 des 22 mâles les. ont chevauchées.

Le comportement copulatoire s'organise dès cette première expérience chez la plupart des animaux.

B. Conditions d'élevage et apparition de l'accouplement

(Epreuves de 5 minutes)

\section{Pourcentage cumulé}

Le pourcentage cumulé de mâles s'étant accouplés au moins une fois reflète à la fois leur motivation et l'efficacité des ajustements posturaux. 
C'est dans le groupe considéré comme témoin (issu d'A.M. et en mixité) que le plus grand nombre de mâles se sont accouplés dans chaque série expérimentale (fig. 3 ).

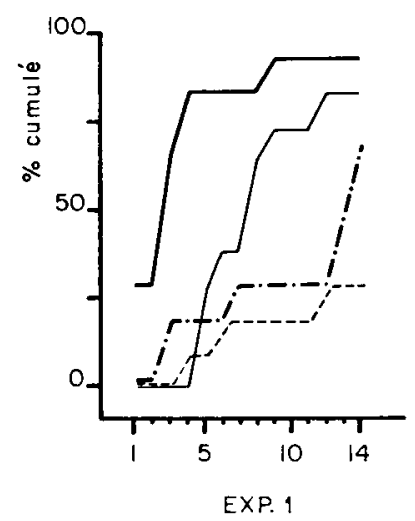

EXP. 1

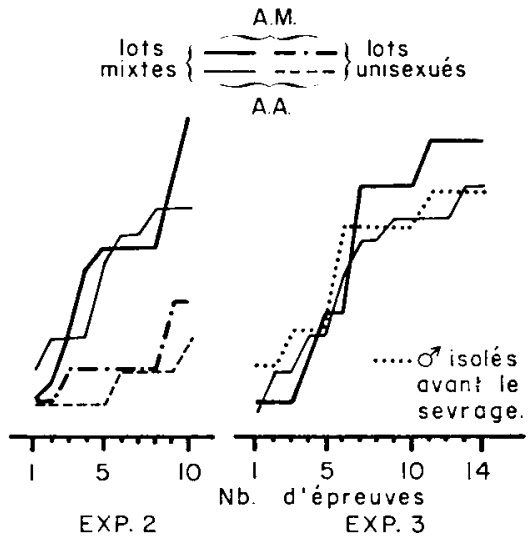

FIG. 3

Apparition et évolution de l'activité copulatoire au cours des épreuves de cinq minutes

(pourcentage cumulé de mâles s'étant accouplés au moins une fois).

Onset and progress of copulatory behaviour in $5 \mathrm{mn}$ sexual behaviour tests (cumulative percentage of males copulating at least once).

La privation du contact avec la mère, avec des femelles du même âge ou même l'isolement dans la période infantile n'affectent pas significativement la proportion de mâles efficaces, si des agnelles sont présentes après le sevrage. Un retard est observé dans le lot mixte issu d'A.A. de l'expérience 1, mais celui-ci est comblé après 8 à 10 épreuves.

Si le contact avec des femelles n'est pas permis dans la période prépubère, la proportion de mâles qui ne s'accouple pas reste significativement supérieure à celle observée dans les groupes mixtes, même si le contact antérieur avec la mère et des jeunes femelles a été assuré dans la période infantile.

De plus, l'apparition du comportement copulatoire est plus rapide dans les groupes mixtes que dans les unisexués $(\mathrm{P}<0,001)$.

\section{Fréquence de monte et d'accouplement des mâles actifs}

Pour cette analyse, nous avons pris en considération les données recueillies à partir du premier accouplement de chaque animal actif.

Pour les montes comme pour les accouplements, les différences de fréquence entre groupes sont peu importantes.

Ainsi, lorsque les jeunes béliers se sont accouplés une fois, la fréquence de montes est peu influencée par les conditions d'élevage. Toutefois, elle est légèrement inférieure pour les animaux du groupe témoin des 2 premières séries expérimentales (fig. 4). De 
même, la fréquence des accouplements, bien que différente entre certains groupes n'est pas modifiée de manière importante par l'environnement social, mais elle est, en particulier dans la première série expérimentale, supérieure pour les mâles du groupe témoin (fig. 5).

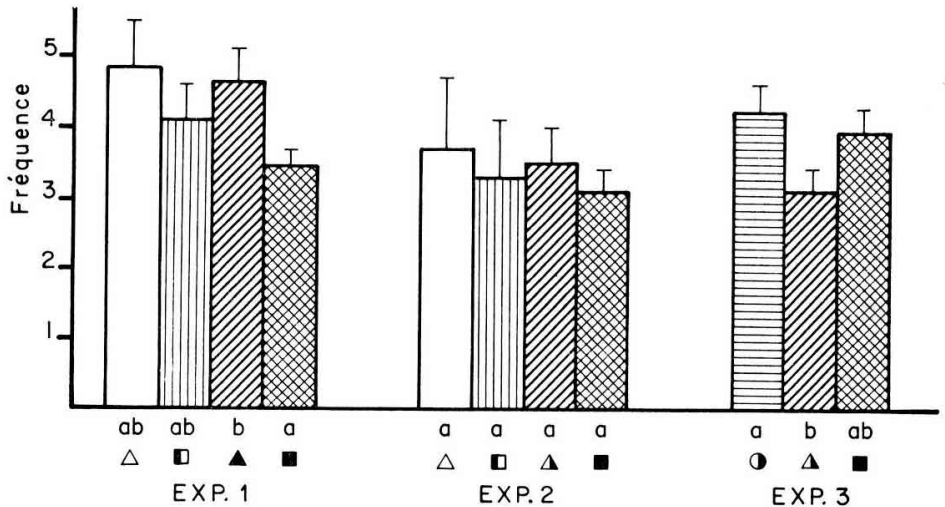

FIG. 4

Fréquence moyenne par mâle actif, et par épreuve, des montes observées pendant les épreuves de cinq minutes

(fig. 4-5-6-7 : les lots désignés par des lettres différentes - a ou bsont statistiquement différents).

Average frequency per active ram and per test of mounts in $5 \mathrm{mn}$ sexual behaviour tests

(fig. 4-5-6-7 : different letters - $a$ or $b-$ indicate statistically significant differences).

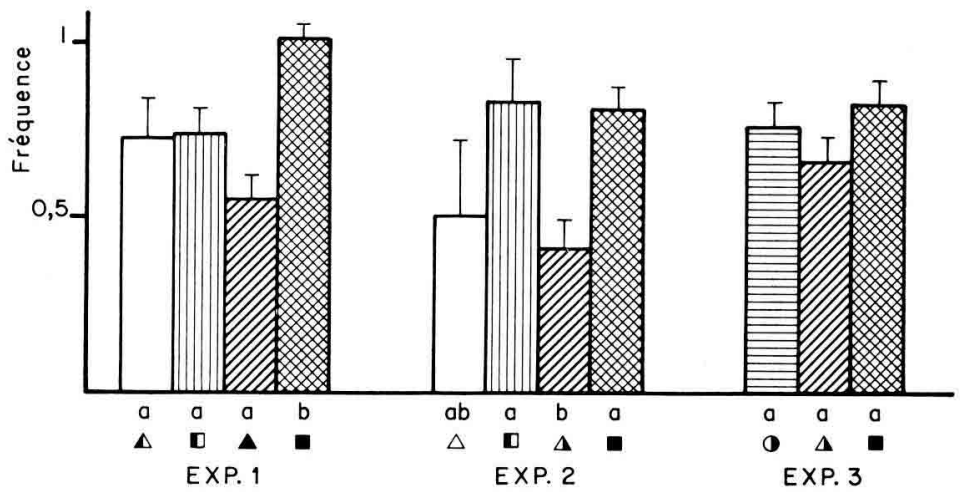

FIG. 5

Fréquence moyenne par mâle actif, et par épreuve,

d'accouplements observés pendant les épreuves de cinq minutes.

Average frequency per active ram and per test of matings in $5 \mathrm{mn}$ sexual behaviour tests.

Il n'est pas possible d'en conclure que la privation d'un contact hétérosexuel dans la période prépubère soit très préjudiciable à l'intensité de l'activité copulatoire dès lors que celle-ci est apparue. A l'inverse, ce contact hétérosexuel n'est pas bénéfique dans tous les cas sur la fréquence des actes sexuels. 
3. Efficacité sexuelle des mâles actifs

Dans les deux premières expériences, nous l'avons vu, les mâles du groupe témoin chevauchent en moyenne moins que les autres pour aboutir à un accouplement. Cela se traduit par une efficacité moyenne significativement supérieure des mâles de ce groupe sur ceux des 3 autres (fig. 6 a).
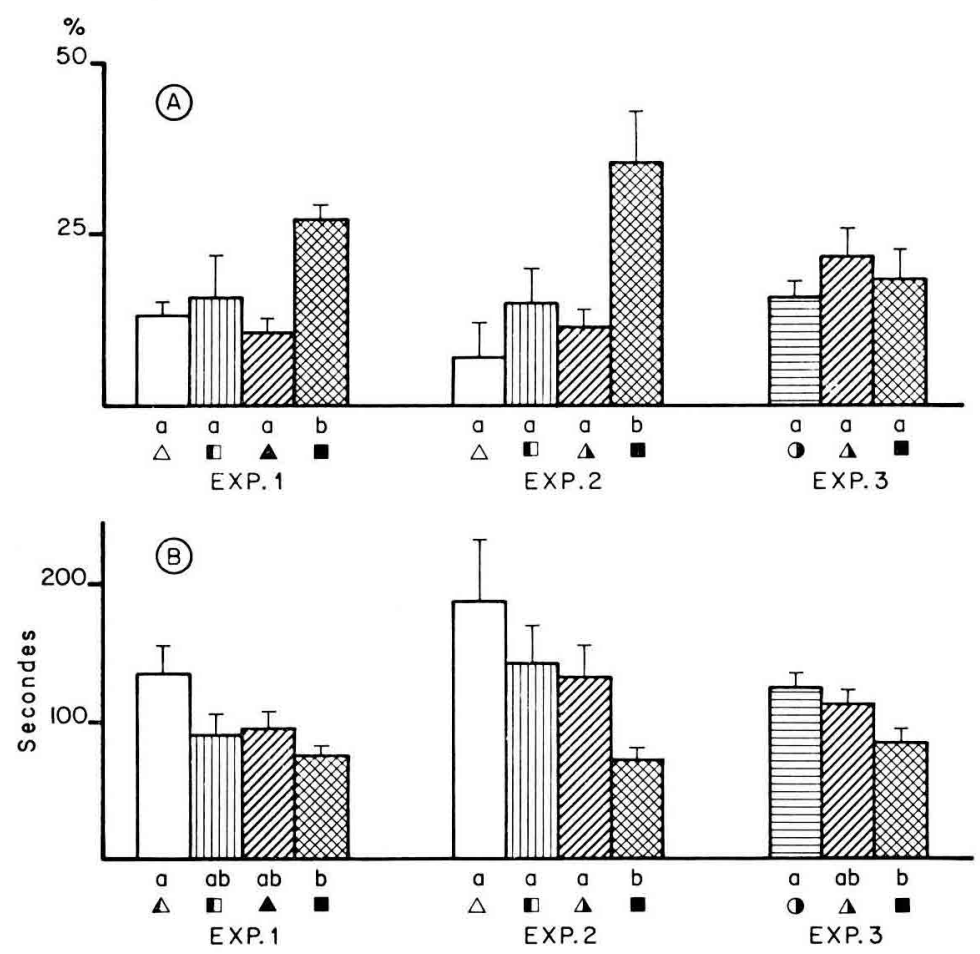

FIG. 6

Efficacité moyenne par mâle actif $(A)$

et latence moyenne en secondes $(B)$ au premier accouplement par mâle actif et par épreuve au cours des épreuves de cinq minutes.

Average sexual efficiency per active ram $(A)$

and latency to first mating in seconds $(B)$ in $5 \mathrm{mn}$ sexual behaviour tests.

Cependant, la privation de la mère en plus de celle d'un contact avec des jeunes femelles ou l'isolement (A.A. groupés et isolés avant le sevrage), ne sont pas préjudiciables si des femelles sont présentes au-delà du sevrage ( $3^{*}$ série expérimentale).

La latence moyenne au premier accouplement est en moyenne plus courte pour les jeunes béliers du groupe témoin (fig. 6 b). Cependant, la différence n'est pas toujours significative, elle l'est principalement avec les mâles qui sont privés de la mère dans la période infantile puis de femelles dans la période prépubère. 
4. Relation entre jeu sexuel et comportement sexuel adulte

D'une part, il n'existe aucune corrélation significative entre un « jeu sexuel » postnatal intense (Orgeur \& Signoret, sous presse) et une apparition précoce de l'activité copulatoire.

D'autre part, il n'y a pas non plus de corrélation entre la fréquence d'activité juvénile de type sexuel et celle d'activité sexuelle adulte (test de corrélation par rang de Spearman).

\section{Conditions d'élevage et capacité sexuelle (Epreuves de 1 heure)}

\section{Proportion de mâles s'étant accouplés au moins une fois}

La majeure partie des béliers a acquis la capacité de réaliser un accouplement et les différences entre lots expérimentaux ne permettent plus avec ce type d'analyse de mettre en évidence d'effet des privations sociales (fig. 7 a).
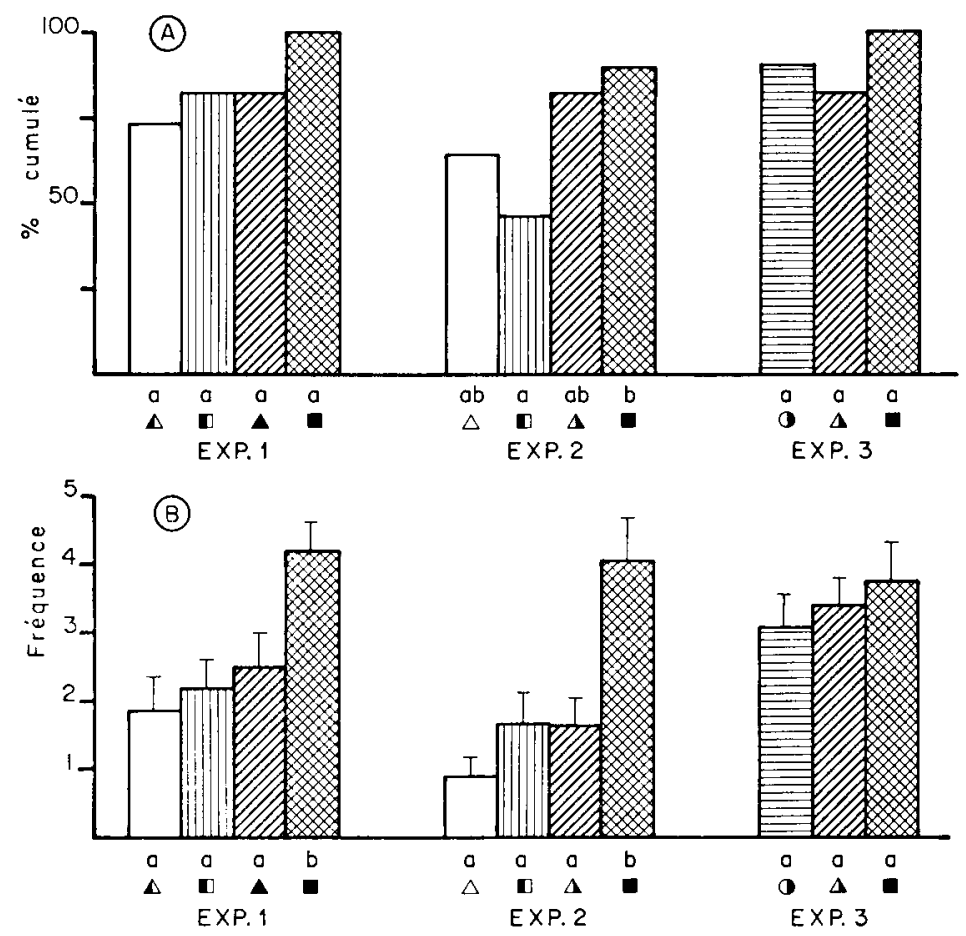

FIG. 7

Pourcentage cumulé de mâles s'étant accouplés au moins une fois $(A)$ et fréquence moyenne par mâle et par épreuve, d'accouplements (B) observés au cours des épreuves d'une heure.

Cumulative percentage of males copulating at least once $(A)$ and average frequency of mating per male and per test $(B)$ during one hour sexual behaviour tests. 
2. Fréquence moyenne d'accouplement (fig. $7 \mathrm{~b}$ )

Elle est significativement plus élevée dans le groupe témoin des 2 premières séries expérimentales, bien que la proportion de mâles actifs ne soit pas supérieure. Ils sont donc plus efficaces dans ce groupe.

Cependant, dans l'expérience 3, aucun effet dû aux privations de la mère et de femelles ou à l'isolement avant le sevrage n'apparaît.

\section{Possibilités d'utilisation pour l'insémination artificielle}

Outre leur aspect pratique, les essais de collecte de semence ont présenté un intérêt théorique, ils révèlent la faculté d'adaptation des mâles à une situation nouvelle de comportement et reflètent leur émotivité.

La proportion de mâles qui ont pu être collectés est inférieure dans les 2 premières séries expérimentales à celle des mâles qui s'étaient accouplés. Une exception concerne le groupe témoin de l'expérience 2 soumis à une technique d'entraînement plus intensive (fig. 8).

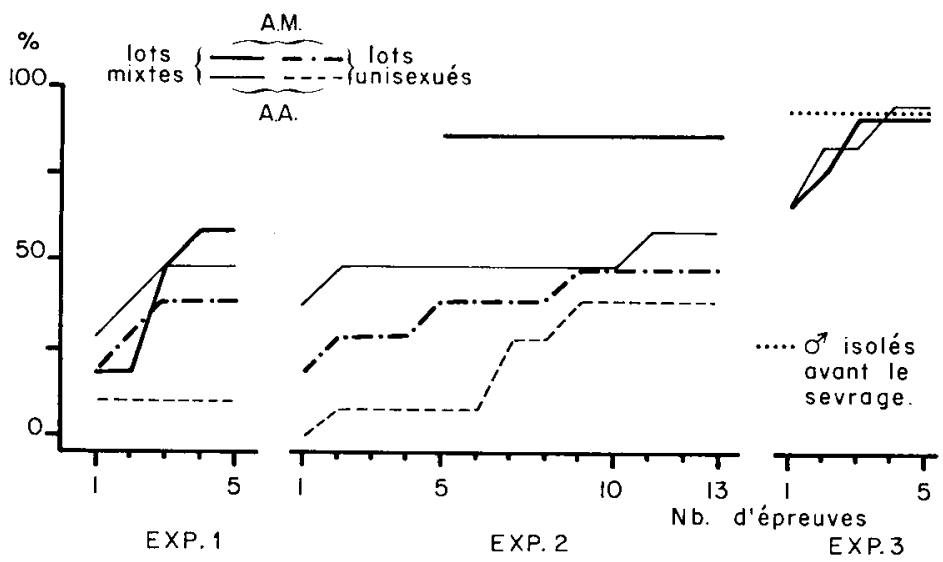

Fig. 8

Evolution de la proportion des mâles

sur lesquels a été effectuée au moins une collecte de sperme.

Proportion of rams having ejaculated at least once in semen collection tests.

Globalement, cette proportion est supérieure dans les lots mixtes ; mais la différence avec ceux qui sont privés de femelles dans la période prépubère n'est pas toujours significative. Elle reste cependant la plus faible dans le groupe unisexué privé antérieurement de la mère (mâles A.A. des 2 premières expériences). 
Il est également important de signaler que les jeunes béliers les plus efficaces au cours des épreuves de 5 minutes et d'une heure, le restent lors des essais de collecte de semence.

\section{Discussion - Conclusion}

Les résultats des 3 séries expérimentales, portant sur 125 jeunes béliers mettent en évidence une influence des conditions d'élevage sur l'apparition et l'évolution de l'activité sexuelle plus que sur son niveau.

Le contact avec des femelles lors de la période prépubère (entre 3 mois et demi et 6 mois environ) influe favorablement sur lapparition de l'activité copulatoire des jeunes béliers et sur son évolution dans le temps.

La privation d'un contact hétérosexuel dans cette période aboutit à un déficit notable qui se traduit par un retard souvent important dans le démarrage de l'accouplement.

La fréquence des actes sexuels, en particulier celle des accouplements est peu affectée, dès lors qu'un premier accouplement a eu lieu.

Ces résultats sont en accord avec ceux publiés par divers auteurs pour qui l'importance des perturbations varie néanmoins. Ainsi selon Hulet et al. (1964), 17 p. 100 de béliers inhibés sont encore inactifs après 8 ou 9 jours d'association avec des femelles en cestrus. Pour Pretorius (1967), jusquà 30 p. 100 de sujets privés de contact avec des femelles de la naissance à la première utilisation, ne présentent toujours pas d'intérêt pour des brebis en chaleur après 38 présentations.

D'autres auteurs ont montré que l'avenir comportemental du jeune bélier est influencé par les relations sociales au cours du développement. C'est le cas de Banks (1964), Marincowitz et al. (1966), Dyrmundsson \& Lees (1972), Le Roux \& BarNARD (1974), ZenchaK et al. (1977, 1980, 1981), Alberio (1976), Rouger (1974, résultats non publiés), WINFIELD \& MAKIN (1978). Toutefois, certaines études ne confirment pas la présence d'anomalies comportementales importantes dues aux conditions d'élevage (Bryant, 1975 ; Fletcher, 1976; Illius et al., $1976 \mathrm{a}, \mathrm{b}$; Welch \& Kilgour, 1978, résultats non publiés : in Wodzicka-TomaszewsKa et al., 1981).

Le mécanisme de mise en route de la réponse sexuelle est plus perturbé que l'efficacité sexuelle, c'est-à-dire que l'organisation posturale est moins affectée que le déclenchement de l'activité sexuelle.

Cependant, la présence de femelles dans la période prépubère n'est peut-être pas le seul facteur important. Nos résultats suggèrent que la présence de la mère lors de la période infantile renforce l'influence favorable ultérieure de jeunes femelles. Cet effet apparaît dans 2 séries expérimentales sur 3, notamment sur l'efficacité sexuelle et dans une moindre mesure, sur la proportion de mâles actifs et la fréquence des actes sexuels. Toutefois, l'absence de la mère ne s'est pas révélée préjudiciable dans la troisième série expérimentale, aussi ne pouvons-nous pas conclure qu'elle entraîne dans tous les cas un déficit comportemental important, au moins dans la mesure où des femelles sont présentes ultérieurement. 
De plus, sa présence est insuffisante si elle est suivie d'une ségrégation des sexes après le sevrage.

Par ailleurs, la privation d'un contact hétérosexuel limitée à la période infantile n'est pas déterminante sur l'avenir sexuel des jeunes mâles. De même, l'élevage en mixité dans cette première période de vie, n'est pas bénéfique s'il est suivi d'une ségrégation sexuelle.

Enfin, l'isolement social partiel pendant les 3 premiers mois de la vie n'a pas de conséquence défavorable sur l'apparition, l'évolution et le niveau de l'activité copulatoire d'animaux entretenus en présence de femelles dans la phase prépubère.

Cela est en accord avec les observations de ANDERson \& ZenchaK (1976) et ZENCHAK (1977), selon lesquelles de jeunes ovins mâles sans expérience de contact avec des congénères pendant les 5 premiers mois de leur vie se comportent normalement en situation hétérosexuelle, alors que s'ils ont eu une expérience sociale en groupes unisexués, ils présentent dans la même situation un comportement de type homosexuel.

Au cours du développement, et dès les premiers jours de la vie, le jeune agneau présente toutes les manifestations motrices et posturales du comportement sexuel mâle. $\mathrm{Ce}$ 《jeu sexuel », particulièrement intense dans les premières semaines de la vie (ORGEUR \& SIgNORET, sous presse), pourrait intervenir dans l'organisation des conduites de l'adulte. Cette hypothèse n'est pas confirmée ici. En effet, 24 heures environ après la réunion des mâles élevés en cases individuelles, les postures sexuelles inadaptées, comme les montes inversées ou perpendiculaires, les parades dirigées de manière aberrante ont pratiquement disparu, alors qu'elles sont très fréquentes sitôt après le rassemblement des animaux.

La récupération d'un comportement «normal » intervient dans nos conditions beaucoup plus rapidement que dans certaines espèces comme le rat (WILHELmSSON \& LARSSON, 1973), le chien (BEACH, 1968), le macaque (HARLow et al., 1972) ou le coq domestique (VIDAL, 1975).

Toutefois, Wood-Gush (1958) chez le coq, BEACH $(1942$, 1958) chez le rat, Bryant (1975) chez les ovins ont déjà montré que les effets de l'isolement ne sont pas toujours très marqués, ni irréversibles.

De plus, rappelons qu'il n'existe pas de relation entre les conduites sexuelles juvénile et adulte.

Ainsi notre étude permet-elle de conclure que dans l'espèce ovine, l'impact d'un contact hétérosexuel précoce sur la mise en place et le déroulement des conduites sexuelles n'est pas limité à une brève période critique post-natale, aux effets irréversibles, comme Lorenz (1935), Schutz (1965), Hess (1973) l'ont affirmé chez les oiseaux.

Si le contact avec la mère dans la période la plus précoce ne paraît pas négligeable, comme le suggèrent les résultats de Rouger (1974, résultats non publiés) et d'Alberio (1976), les facteurs influençant la manifestation du comportement sexuel semblent plutôt intervenir au cours d'une phase plus tardive du développement. L'avenir de reproduction du jeune bélier est en effet favorablement influencé par un contact hétérosexuel prépubère, malgré le faible niveau d'activité sexuelle existant dans cette période. C'est à ce moment-là que le jeu sexuel devient progressivement du comporte- 
ment sexuel avec un choix préférentiel du sexe du partenaire et une organisation séquentielle des actes comportementaux très marqués.

De nombreux auteurs : Hulet (1966), Marincowitz et al. (1966), Mattner et al. (1971 a), Dyrmundsson \& Lees (1972), Zenchak et al. (1977, 1980, 1981), AlbeRIO (1976), Rouger (1974, résultats non publiés) avaient mentionné, mais sans préciser la phase importante, l'effet bénéfique d'un contact hétérosexuel au cours du développement.

Par contre, Banks (1964), Pretorius (1967), Le Roux \& Barnard (1974), WinFIELD \& MAKIN (1978) ont suggéré comme nous le rôle primordial de la période prépubère.

Cette période correspond à la phase de croissance testiculaire rapide (déjà amorcée vers la $8^{\circ}$ semaine), reflet de la mise en place des phénomènes de spermatogenèse. C'est au cours de cette phase que la sécrétion de testostérone s'accrô̂t et que les premiers spermatozoïdes apparaissent dans les tubes séminifères (COUROT, 1962).

Cependant, nous n'avons pas mis en évidence de relation entre la taille testiculaire ou le poids corporel et l'activité sexuelle.

Enfin, la majorité des mâles qui n'ont pas montré d'activité sexuelle ou qui n'ont pu s'accoupler au cours des différentes mises à l'épreuve, sont devenus actifs lors de leur utilisation en élevage (information non quantifiée, recueillie auprès des responsables de l'élevage). Ceci confirme les observations faites par Hulet et al. (1964), LE Roux \& Barnard (1974), ANDERson \& ZenchaK (1976) selon lesquelles des jeunes béliers inhibés en situation hétérosexuelle, deviennent efficaces avec l'expérience. Toutefois, d'après MATTNER et al. (1971 b), leur niveau de performance reste inférieur à celui de mâles qui se sont accouplés d'emblée.

De ce fait, le retard dans le démarrage de l'activité copulatoire suivi d'un niveau de performance insuffisant, peut avoir d'importantes conséquences économiques.

\section{Summary}

Effect of the social environment during development on the onset and intensity of sexual behaviour in young sheep

In many mammalian species, the social environment during the development play an important role in the interactions between adults, especially in the sexual behaviour. In domestic species, management techniques may lead to some social deprivations.

The aim of this study was to examine the effect of the following situations on the sexual behaviour of domestic sheep :

1) artificial rearing in the absence of the dam, sexual segregation and social isolation during the first 3 months of life from birth to weaning;

2) rearing in contact with age-mate females during the prepuberal period from weaning to puberty (6 months of age).

Three experimental series involving a total of 125 male lambs of the Ile-de-France breed (born in February) were tested for the development of sexual behaviour from the age of 6 months (fig. 1). 
The first contact of inexperienced ram lambs with oestrus females considerably stimulated their sexual interactions (fig. 2). When presented individually to oestrus females in a $5 \mathrm{mn}$ sexual behaviour test, the copulatory activity was observed earliest in groups associated with females before puberty. The absence of contact with females or with the dam, and even a total isolation before weaning did not affect the subsequent display of copulatory activity (fig. 3).

Once initiated, the copulatory activity was no longer influenced by the early experience (fig. 4 and 5). The sexual activity (fig. 6 a) was higher and the mating latency shorter (fig. $6 \mathrm{~b}$ ) in males reared by their dam and in mixed groups from birth to puberty.

The frequency of prepuberal sexual activity was not correlated either with the earliness or with the intensity of adult copulatory behaviour. However, in the first two experimental series, the sexual capacity of the young rams was higher in those reared by their dam and kept in mixed groups (fig. $7 \mathrm{~b}$ ). The percentage of active rams obtained in one-hour-tests with several oestrus females was not affected by the treatments (fig. $7 \mathrm{a}$ ).

Semen collection results were similar although somewhat lower than those obtained with natural mating. The percentage of rams in which semen could be collected was lower in dam and female deprived males (fig. 8).

It may be concluded that preweaning conditions (absence of the dam, sexual segregation or social isolation) had no detrimental effect on the development of copulatory activity, provided that the males were in contact with females during the prepuberal period.

Key words : Sheep, male sexual behaviour, development, social environment, puberty.

Reçu en avril 1983.

Accepté en décembre 1983.

\section{Références bibliographiques}

Alberio R., 1976. Rôle de la photopériode dans le développement de la fonction de reproduction chez l'agneau lle-de-France de la naissance à 21 mois. Thèse Doct. $3^{*}$ cycle, Paris, 57 p.

Anderson G.C., ZenchaK J.J., 1976. Homosexual behavior in rams (Ovis aries) and rearing experience. Proc. 8th Int. Cong. Anim. Reprod. and I.A., Krakow, 1196.

BanKs E., 1964. Some aspects of sexual behavior in domestic sheep (Ovis aries). Behaviour, 23, 249-279.

BEACH F.A., 1942. Comparison of copulatory behavior of male rats raised in isolation, cohabitation and segregation. J. Genetic Psychol., 60, 121-136.

BEACH F.A., 1958. Normal sexual behavior in male rats isolated at fourteen days of age. J. Comp. Physiol. Psychol., 51, 37-38.

BEACH F.A., 1968. Coital behaviour in dogs. III. Effects of early isolation on mating in males. Behaviour, 30, 218-238.

BRyanT M.J., 1975. A note on the effect of rearing experience upon the development of sexual behavior in ram lambs. Anim. Prod., 21, 97-99.

Courot M., 1962. Développement du testicule chez l'agneau. Etablissement de la spermatogenèse. Ann. Biol. anim. Biochim. Biophys., 2, 25-41.

Dyrmundsson O.R., LeEs J.L., 1972. Effect of rams on the onset of breeding activity in Clun Forest ewe lambs. J. Agric. Sci. Camb., 79, 269-271.

FABRE C., 1977. Etude du comportement sexuel mâle induit par traitement aux hormones stéroïdes chez la brebis adulte ovariectomisée. Thèse $3^{\circ}$ cycle, Univ. Paris VI, France, $66 \mathrm{p}$.

Fletcher I.C., 1976. Sexual activity in Merino rams : in Sheep breeding «G.T. Tomes, D.E. Robertson and R.J. Lightfood » (ed.). Proc. Int. Congr. Muresk and Perth, W.A., Aug. 1976, 345-351. 
Harlow H.F., Harlow M.K., Hansen E.W., Suomi S.J., 1972. Infantile sexuality in monkeys. Arch. Sex. Behav., 2, 1-7.

Hess E.H., 1973. Imprinting. Early experience and the developmental psychobiology of attachment. Van Nos. - Reinhold $\mathrm{C}^{\circ}$ Ed., New York.

Hulet C.V., 1966. Behavioural, social and psychological factors affecting mating time and breeding efficiency in sheep. J. Anim. Sci., 25, Suppl., 5-20.

Hulet C.V., Blackwell R.L., Ercanbrack S.K., 1964. Observations on sexually inhibited rams. J. Anim. Sci., 23, 1095-1097.

Illius A.W., Haynes N.B., Lamming G.E., 1976 a. Effects of ewe proximity on peripheral plasma testosterone levels and behaviour in the ram. J. Reprod. Fert, 48, 25-32.

Illius A.W., Haynes N.B., Purvis K., Lamming G.E., 1976 b. Plasma concentrations of testosterone in the developing ram in different social environments. $J$. Reprod. Fert., 48, 17-24.

Le Roux P.J., Barnard J.P., 1974. The effect of heterosexual contact on libido and mating dexterity in Karakul rams. S. Afr. J. Anim. Sci., 4, 171-174.

LoRenZ K., 1935. Der Kumpan in der Umwelt des Vogels. J. Ornithol., 83, 137-213, 289-413.

Marincowitz G., Pretorius P., Herbst S., 1966. Dominance in sexual behaviour and mating dexterity in merino rams. S. Afr. J. Agric. Sci., 9, 971-980.

Mattner P.E., Braden A.W.H., George J.M., 1971 a. Studies in flock mating of sheep. 4. The relation of libido tests to subsequent service activity of young rams. Aust. J. Exp. Agric. Anim. Husb., 11, 473-477.

Mattner P.E., Braden A.W.H., George J.M., 1971 b. Incidence and duration of sexual inhibition in young rams. J. Reprod. Fert., 24, 149-150.

Mattner P.E., Braden A.W.H., George J.M., 1973. Studies in flock mating of sheep. 5. Incidence, duration and effect on flock fertility of initial sexual inactivity in young rams. Aust. J. Exp. Agric. Anim. Husb., 13, 35-41.

Orgevr P., Signoret J.P. The sexual play and its functional significance in the domestic sheep (Ovis aries L.). Physiol. Behav. (sous presse).

Pretorius P.S., 1967. Libido and mating dexterity in rams reared and kept in isolation from ewes. Proc. S. Afr. Soc. Anim. Prod., 6, 208-212.

RoBinson T.J., 1954. Relationship of oestrogen and progesterone in oestrous behaviour of the ewe. Nature, Lond., 173, 878.

Schutz F., 1965. Sexuelle Prägung bei Anatiden. Z. Tierpsychol., 22, 50-103.

SIEGEL S., 1956. Non parametric statistics for the behavioral sciences. Mc Graw-Hill, New York.

VIDaL J.M., 1975. Influence de la privation sociale et de l' «autoperception» sur le comportement sexuel du coq domestique. Behaviour, 52, 57-83.

Wilhelmsson M., Larsson K., 1973., The development of sexual behavior in anosmic male rats reared under various social conditions. Physiol. Behav., 11, 227-232.

Winfield C.G., Makin A.W., 1978. A note on the effect of continuous contact with ewes showing regular oestrus and of post-weaning growth rate on the sexual activity of Corriedale rams. Anim. Prod., 27, 361-364.

Wodzicka-Tomaszewska M., Kilgour R., Ryan M., 1981. "Libido» in the larger farm animals : a review. Appl. Anim. Ethol., 7, 203-238.

Woon-Gush D.G.M., 1958. The effect of experience on the mating behaviour of the domestic cock. Anim. Behav., 6, 68-71.

ZENCHAK J.J., 1977. Early socialization effect on adult ram sexual behavior. Ph. D. Thesis. West Virginia University.

ZenchaK J.J., Anderson G.C., 1980. Sexual performance levels of rams (Ovis (uries) as affected by social experiences during rearing. J. Anim. Sci., 50, 167-174.

Zenchak J.J., Anderson G.C., Schein M.W., 1981. Sexual partner preference of adult rams (Ovis aries) as affected by social experiences during rearing. Appl. Anim. Ethol., 7, 157-167. 